\title{
VALORES SINGULARES DE MATRIZES UNIFORMES
}

Antonio Francisco Iemma

Departamento de Matemātica e Estatística. Escola Superior de Agricul tura "Luiz de Queiroz", USP - 13.400 - Piracicaba, SP.

\section{RESUMO}

Apresenta-se um procedimento simples e direto para a obten ção de. valores singulares de matrizes uniformes definidas não nega tivas, através do uso das matrizes de Helmert. Esse procedimento é sugerido aos professores de cursos iniciais em estatística ofereci dos a alunos com pequenos conhecimentos em algebra de matrizes.

\section{SUMMARY}

IEMMA, A.F. The singular value decomposition of a uniform matrix. 1982. Ciência e Natura (4):21-26.

In this paper is presented a simple procedure for determi nation of singular value of uniform non negative matrices. This pro cedure is recommended for teachers of elementary courses in statis tics to students with small basis in matrix algebra.

\section{INT RODUÇAO}

Os valores singulares de matrizes têm sido fundamentais pa ra a demonstração de muitos dos teoremas relacionados com a estatís tica. Conforme pode ser constatado em BOX (2), GRAYBILL (4), SCHEFFE (9) e SEARLE (10), dentre outros, uma importante aplicação dos valo res singulares é feita no estudo das formas quadrāticas associadas aos quadrados médios utilizados na anālise de variāncia. Sabe-se da teoria geral, por exemplo, que sob certas condições, uma soma de quadrados do resíduo pode ser escrita como $S Q R=\sum \lambda_{j} \chi_{i}^{2}:(p)$, onde $\lambda_{j}$ são valores singulares positivos da matriz de covariâncias $v$ e $x_{j}^{2}$ (p) são qui-qua drados centrais com p graus de liberdade. Nesse contexto, torna-se desnecessário ressaltar a importância dos valores singulares. No en tanto, sua determinação geralmente é trabalhosa e muitas vezes re quer o uso de computadores. Neste artigo é apresentado um processo simples e direto de obtenção dos valores singulares de matrizes de covariāncias do tipo uniforme, atravēs do uso das matrizes de Helmert.

\section{DESENVOLVIMENTO TEORICO}

Segundo GEISSER (3), uma matriz uniforme de covariāncia, é do tipo

$$
V=\left[\begin{array}{cccc}
\sigma^{2} & \rho \sigma^{2} & --- & \rho \sigma^{2} \\
\rho \sigma^{2} & \sigma^{2} & --- & \rho \sigma^{2} \\
\hdashline \sigma^{2} & \rho \sigma^{2} & --- & --- \\
-\sigma^{2}
\end{array}\right]
$$


onde $\sigma^{2}$ e $\rho$ são respectivamente a variancia e o coeficiente de cor relação constante.

Em situações experimentais a identificação das estimativas $\hat{V}$ das matrizes uniformes é feita atravēs dos testes de homogeneida de e uniformidade como, por exemplo, aqueles de WILKS (11) e BoX (1).

Matrizes de covariāncias desse tipo são de grande utilida de, mormente nos ensaios que sugerem "split-plot", no tempo, para os quais a condição de uniformidade pode indicar a alternativa mais correta de anālise dos dados, entre um procedimento univariado usual e um procedimento multivariado como a anālise de perfil.

SEARLE (10), com base nas idéias de LANCASTER (7), define a matriz de Helmert, de ordem $n$, por:

$$
H(n)=\left[\begin{array}{c}
h^{\prime} \\
-- \\
H
\end{array}\right]
$$

onde $h^{\prime}$ é um vetor linha de $n$ componentes iguais a $\frac{1}{\sqrt{n}}$ :

$$
h^{\prime}=\left[\frac{1}{\sqrt{n}}, \frac{1}{\sqrt{n}}, \ldots, \frac{1}{\sqrt{n}}\right]
$$

e $H$ é uma matriz de $(n-1)$ linhas e $n$ colunas, na qual a i-ésima li nha è obtida de

$$
\left[\frac{1}{\sqrt{r(r+1)}} p^{\prime}(r) ; \frac{-r}{\sqrt{r(r+1)}} ; \phi(n-r-1) \times(1)\right] ; r=1,2, \ldots, n-1
$$

sendo $\mathrm{p}^{\prime}(r)$ um vetor linha com $r$ componentes iguais a 1 .

Notadamente as matrizes de Helmert são ortogonais e sus tentam as propriedades necessárias para a decomposição ortogoną de matrizes definidas não negativas.

Por outro lado, um processo amplamente abordado na litera tura, e que tem sido usado em alguns "pacotes" computacionais, para a obtenção de valores singulares consiste na resolução da equação caracterîstica:

$$
\delta(\bar{V}-\lambda I)=0
$$

onde $\delta$ é 0 determinante, V̄é uma estimativa da matriz de covariâncias, $\lambda \bar{e}$ o vetor dos valores singulares desconhecidos e I é uma matriz identidade com as mesmas dimensões de $\hat{v}$. Esse processo pode apresen tar-se inconvenientemente quando $\delta$ for de ordem elevada.

A decomposição por valores singulares pode apresentar os mesmos inconvenientes, se na obtenção da matriz ortogonal $P$, tal que

$$
P^{\prime} \hat{V} P=\operatorname{diag}\left\{\lambda_{1}, \lambda_{2}, \ldots, \lambda_{k}\right\}
$$

onde $\lambda_{j}(j=1,2, \ldots, k)$ 'são os valores singulares, for usado a 1 
gum processo que envolva determinantes.

Considerando-se o estreito relacionamento existente entre as matrizes ortogonais $P$ e as matrizes $H(n)$ de Helmert, propõe-se aqui a substituição de $P$ por $H^{\prime}(n)$, em $(\alpha .4)$, resultando:

$$
H_{(n)} \hat{V} H^{\prime}(n)=\operatorname{diag}\left\{\lambda_{1}, \lambda_{2}, \ldots, \lambda_{k}\right\} \quad(\alpha .5)
$$

Naturalmente a uniformidade de $\hat{V}$ garante sua simetria. Por outro la do, considerou-se neste estudo apenas o caso em que $\bar{v} \bar{e}$ positiva dé finida.

Como as matrizes $H(n)$ apresentam uma consistente lei de formação, $(\alpha .2)$, que depende apenas de $n$, então elas podem ser fá cilmente obtidas, tornando imediato o processo de obtenção dos valo res singulares. Uma identificação mais imediata de $H_{(n)}$ pode ser fei ta em $(\alpha .6)$. Assim, efetuando-se os produtos sugeridos em $(\alpha .5)$ ve rifica-se que $\hat{V}$ tem dois valores singulares:

$$
\left.\lambda_{1}=[1+(n-1) \hat{\rho}] \hat{\sigma}^{2} \text { e } \lambda_{2}=(1-\hat{\rho}) \bar{\sigma}^{2} \text {, (multiplicidade }=n-1\right)
$$

Desse modo, se a matriz de covariância é do tipo uniforme tem-se aqui, dois critērios para a obtenção de seus valores singula res, atravēs de $(\alpha .5)$ ou $(\alpha .7)$ :

$$
\text { (a.6) }
$$

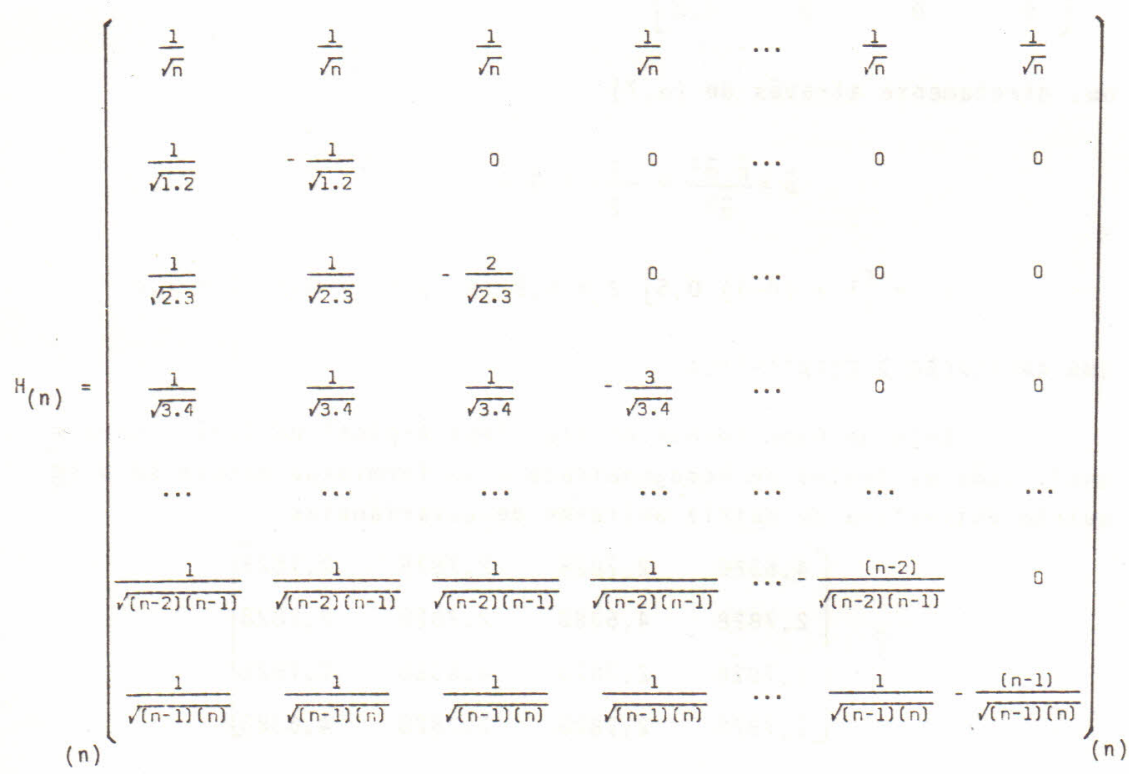


APRESENTAÇAO DE UM EXEMPLO

Seja a matriz uniforme $A$, de dimensão 4 , onde

$$
a_{i j}=\left\{\begin{array}{l}
2, \text { se } i=j \\
1, \text { se } i \neq j
\end{array}\right.
$$

os valores singulares de $A$, assim definida, podem ser obtidos facil mente de $(\alpha .5)$ :

$$
H_{(4)} A H^{\prime}(4)=\operatorname{diag}\left\{\lambda_{1} \lambda_{2}\right\} \Longrightarrow
$$

$\Rightarrow\left[\begin{array}{cccc}\frac{1}{\sqrt{4}} & \frac{1}{\sqrt{4}} & \frac{1}{\sqrt{4}} & \frac{1}{\sqrt{4}} \\ \frac{1}{\sqrt{2}} & -\frac{1}{\sqrt{2}} & 0 & 0 \\ \frac{1}{\sqrt{6}} & \frac{1}{\sqrt{6}} & -\frac{2}{\sqrt{6}} & 0 \\ \frac{1}{\sqrt{12}} & \frac{1}{\sqrt{12}} & \frac{1}{\sqrt{12}} & -\frac{3}{\sqrt{12}}\end{array}\right]\left[\begin{array}{cccc}2 & 1 & 1 & 1 \\ 1 & 2 & 1 & 1 \\ 1 & 1 & 2 & 1 \\ 1 & 1 & 1 & 2\end{array}\right]\left[\begin{array}{cccc}\frac{1}{\sqrt{4}} & \frac{1}{\sqrt{2}} & \frac{1}{\sqrt{6}} & \frac{1}{\sqrt{12}} \\ \frac{1}{\sqrt{4}} & -\frac{1}{\sqrt{2}} & \frac{1}{\sqrt{6}} & \frac{1}{\sqrt{12}} \\ \frac{1}{\sqrt{4}} & 0 & -\frac{2}{\sqrt{6}} & \frac{1}{\sqrt{12}} \\ \frac{1}{\sqrt{4}} & 0 & 0 & -\frac{3}{\sqrt{12}}\end{array}\right]=$

$=\left[\begin{array}{cccc}5,0 & 0 & 0 & 0 \\ 0 & 1,0 & 0 & 0 \\ 0 & 0 & 1,0 & 0 \\ 0 & 0 & 0 & 1,0\end{array}\right]=\operatorname{diag}\left\{\lambda_{1}=5 ; \lambda_{2}=1:(m=3)\right\}$

ou, diretamente atravēs de $(\alpha .7)$

$$
\begin{gathered}
\hat{\rho}=\frac{\hat{\rho} \hat{\sigma}^{2}}{\hat{\sigma}^{2}}=\frac{1}{2}=0,5 \\
\lambda_{1}=[1+(4-1) 0,5] 2=5,0 \text { e } \lambda_{2}=(1-0,5) 2=1,0
\end{gathered}
$$

UMA APLICAÇÃO A ESTATISTICA

Seja um experimento do tipo "Split-plot" no tempo, para o qual, após os testes de homogeneidade e uniformidade obteve-se a se guinte estimativa.de matriz uniforme de covariâncias:

$$
\hat{v}=\left[\begin{array}{llll}
4,6380 & 2,7828 & 2,7828 & 2,7828 \\
2,7828 & 4,6380 & 2,7828 & 2,7828 \\
2,7828 & 2,7828 & 4,6380 & 2,7828 \\
2,7828 & 2,7828 & 2,7828 & 4,6380
\end{array}\right]
$$


OBS: $I=2$ tratamentos, $J=4$ tempos, $k=6$ repetições.

Sabe-se, conforme IEMMA E CAMPOS (6), dentre outros, que para experimentos desse tipo, a decomposição usual para a anāilise de variāncia ē aquela que consta da Tabela $I$, onde $f_{1}, f_{2}$ e $f_{3}$ são fun ções não negativas dos parâmetros. Desse modo, as somas de quadrados para os residuos (a) e (b) ficam bem determinadas, sem que se efetue a anālise de variāncia:

$$
\begin{aligned}
& S Q \operatorname{Res}(\mathrm{a})=\lambda_{1}=[1+(\mathrm{J}-1) \hat{\mathrm{\rho}}] \hat{\sigma}^{2}=12,9864 \\
& \mathrm{SQ} \operatorname{Res}(\mathrm{b})=\lambda_{2}=(1-\hat{\rho}) \hat{\sigma}^{2}=1,8552
\end{aligned}
$$

TABELA I. DECOMPOSIÇĀO USUAL PARA A ANALLISE DE VARIÂNCIA DE EXPERI MENTOS TIPO "SPLIT-PLOT" NO TEMPO, COM MATRIZ UNIFORME DE COVARIÂNCIAS.

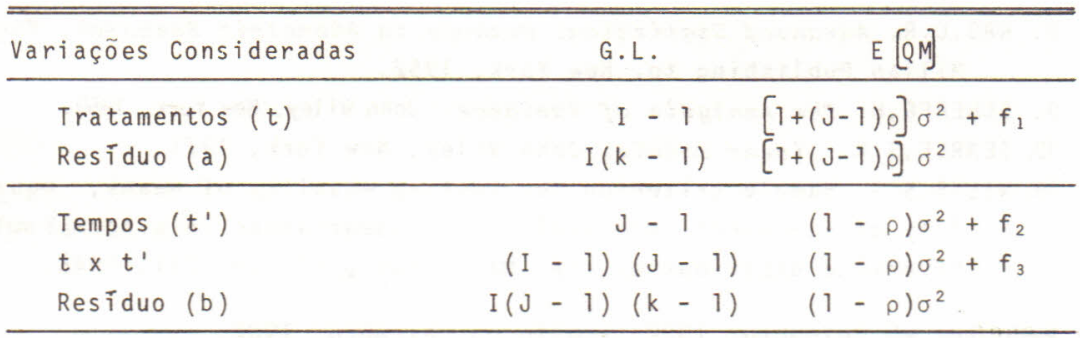

Desse modo, as comparações mūltiplas podem ser efetuadas da simples substituição nas respectivas fórmulas, de SQ Res(a) e SQ Res(b) por $\lambda_{1}$ e $\lambda_{2}$, obtendo-se assim as variâncias desejadas.

\section{CONCLUSṌES}

A decomposição de matrizes simētricas por valores singula res, pode ser muitas vezes trabalhosa e exigir conhecimentos razoá veis sobre ălgebra linear e matricial. Neste estudo verificou-seque se a matriz de interesse é do tipo uniforme, então a simples substị tuição das matrizes ortogonais, obtidas dos autovetores normaliza dos dessa matriz, pelas matrizes de Helmert constitui-se num proces so imediato de obtenção dos valores singulares. Uma aplicação à es tatística à apresentada, enfatizando a importāncia do uso dos valo res singulares e do método proposto.

\section{BIBLIOGRAFIA CITADA}

1. BOX,G.E.P. A general distribuition theory for a class of like lihood criteria. Biometrika, 36: 317-346, 1949.

2. BOX,G.E.P. Some theorems on quadratic forms applied in the study of analysis of variance problems, II. Effects of inequality of variance and correlation between errors in a two way clas 
Sification. Ann. Math. Stat, 25: 484-498, 1954.

3. GEISSER,S. Multivariate analysis of variance for a special case. JASA, 8: 660-669, 1963.

4. GRAYBILL,F.A. Theory and Application of the Linear Models. Duxbury Press, Massachussets, 1976.

5. IEMMA,A.F. Anălise de experimentos em parcelas subdivididas com tratamentos principais dispostos em blocos incompletos balan ceados. ESALQ/USP, Piracicaba, 145 p. (Tese de Doutoramento), 1981.

6. IEMMA,A.F. e H. CAMPOS. Variância dos contrastes clássicos nos ex perimentos com parcelas subdivididas em blocos incompletos ba lanceados. Ciência e Natura, 3: 21-27, 1981.

7. LANCASTER,H.0. The Helmert matrices. Am. Math. Monthly, 72(1): 4-12, 1965.

8. RAO,C.R. Advanced Statistical Methods in Biometric Research. Mac Millan Publishing to, New York, 1952.

9. SCHEFFE,H. The Analysis of Variance. John Wiley, New York, 1959.

10. SEARLE,S.R. Linear Models, John Wiley, New York, 1971.

11. WILKS,S.S. Sample criterion for testing equality of means, equa lity of variances, and equálity of covariances in a normal mul tivariate distribution. Af. Mat. Stat., 17: 257-281, 1946.

Recebido em dezembro, 1982; aceito em dezembro, 1982. 